\title{
A Rare Case of Occipital Stroke as a Consequence of Nonbacterial Thrombotic Endocarditis in Ovarian Clear Cell Carcinoma: A Case Report
}

\author{
S. Devulapalli ${ }^{a} \quad$ N. Pinto $^{b} \quad$ C. Gandothra ${ }^{c}$ \\ A. Jayam-Trouth ${ }^{\mathrm{a}}$ M. Kurukumbi ${ }^{\mathrm{a}}$ \\ Departments of ${ }^{a}$ Neurology, ${ }^{b}$ Medicine and ${ }^{c}$ Cardiology, Howard University \\ Hospital, Washington, D.C., USA
}

\section{Key Words}

Nonbacterial thrombotic endocarditis · Ovarian clear cell carcinoma $\cdot$ Stroke

\begin{abstract}
Hypercoagulability occurs in $15 \%$ of patients with malignancy and represents a clinical spectrum ranging from abnormal coagulation tests but no clinically evident thromboembolic disease, to arterial and venous thrombosis, migratory thrombophlebitis, nonbacterial thrombotic endocarditis (NBTE) and disseminated intravascular coagulation. The combination of increased procoagulant activity and decreased fibrinolytic activity accelerates the prothrombotic potential of endothelial cells in malignancy. NBTE is a rare manifestation of cancer-induced hypercoagulability and is commonly seen with mucinproducing adenocarcinomas, but rarely seen with ovarian clear cell carcinoma (OCCC). Cerebrovascular embolization ranges from $14-91 \%$ in NBTE. We report a rare case of a $62-$ year-old female presenting with occipital stroke as a consequence of NBTE in OCCC. Association of NBTE in OCCC has only been reported in 2 cases so far, but presentation with stroke has never been reported in the literature.
\end{abstract}

\section{Introduction}

Nonbacterial thrombotic endocarditis (NBTE) is a rare manifestation of hypercoagulability in malignancy and very rarely seen in ovarian clear cell carcinoma (OCCC). Systemic embolization to multiple organs secondary to NBTE is common, with embolization to the brain ranging from 14-91\%. Only 2 case reports have shown the 
association between NBTE and OCCC, but we report a rare association of NBTE and OCCC presenting with embolic stroke, which has never been reported in the literature.

\section{Case Report}

A 62-year-old female presented with sudden onset blurry vision on the left side, stating that she was unable to read the hour's portion on a digital clock, but was able to read the minutes. Her medical history includes hypertension and a recent diagnosis of stage I OCCC. At the time of presentation, she was status post-total abdominal hysterectomy and bilateral salpingo-oophorectomy, and had received 6 cycles of chemotherapy with carboplatin and paclitaxel.

After the fifth cycle, she experienced tingling and numbness in all 4 extremities due to paclitaxelinduced peripheral neuropathy, which responded to treatment with pregabalin. She had just finished the sixth cycle of chemotherapy when she presented with this sudden onset of visual complaints. Upon clinical examination, the patient had left homonymous hemianopia, which was confirmed by Humphrey's visual field testing. The rest of the detailed neurologic examination was within normal limits.

Stroke protocol was initiated and MRI of the brain with contrast showed restricted diffusion and bilateral occipital lobe edema, right greater than left, and punctuate foci in the frontal lobes, left caudate and both cerebellar hemispheres, representing acute embolic infarcts ( $\underline{\mathrm{fig}} \mathbf{1} \mathrm{a}, \mathrm{b}$ ). Nonenhancement in $\mathrm{T}_{1}$ post-contrast image of the brain ruled out the possibility of metastases (fig. 1c).

Further stroke workup showed an abnormal lipid profile, with a high-density lipoprotein level of $33 \mathrm{mg} / \mathrm{dl}$ and a low-density lipoprotein level of $157 \mathrm{mg} / \mathrm{dl}$, for which the patient was started on statins. Carotid Doppler ultrasonography and two-dimensional transthoracic echocardiography did not show any abnormalities. Given the suspicion of embolic stroke, a transesophageal echocardiogram (TEE) was obtained, which showed a small mobile linear echodensity (fig. 2a) on the aortic side of the right aortic valve cusp, most consistent with vegetation. Blood cultures done after this finding were negative and the patient was afebrile. There was no evidence of thrombus in the left atrial appendage and there was normal left ventricular function with an intact interatrial septum.

During hospitalization, a CT of the thorax, abdomen and pelvis was done to monitor the progression of OCCC, which showed new metastases to the liver, mesentery and T10 vertebral body (fig. $3 \mathrm{a}, \mathrm{b}$ ). Additionally, an acute pulmonary embolus in the subsegmental arteries of the right lower lung lobe and multiple wedge-shaped infarcts in both kidneys were found (fig. 3c, d). Doppler ultrasonography of the bilateral lower extremities showed deep vein thrombosis (DVT) in the left distal popliteal vein and left peroneal vein. A prophylactic inferior vena cava (IVC) filter was placed. A repeat TEE done after the initiation of anticoagulation revealed a decrease in the size of the vegetation (fig. $2 \mathrm{~b}$ ).

Other confounding factors for stroke, including paroxysmal nocturnal hemoglobinuria marker, protein C, protein S, factor Leiden V, antithrombin III, antinuclear antibody, lupus anticoagulant, erythrocyte sedimentation rate, homocysteine and vitamin B12 levels, were within normal limits. There was no family history of hypercoagulable syndromes. The patient was put on a therapeutic dose of enoxaparin and her vision gradually improved during her hospitalization. Warfarin was not initiated due to its ineffectuality in treating NBTE. The patient was discharged home in stable condition, and follow-up with oncology was ordered for continued management.

Written informed consent was obtained from the patient for publication of this case report and accompanying images. 


\section{Discussion}

In 1994, Chaturvedi et al. [1] retrospectively assessed a cohort of patients with cancer who had cerebral ischemic events in order to determine if the occurrence of the ischemic event was a manifestation of Trousseau's syndrome (spontaneous recurrent or migratory episodes of venous thrombosis, arterial emboli due to nonbacterial thrombotic endocarditis, or both in malignant neoplasms). Based on their study, they concluded that ischemic cerebral events in cancer patients should not serve as sufficient evidence of Trousseau's syndrome until prospective studies were conducted to evaluate the role of hypercoagulability in cancer-related strokes. Since then, extensive research and numerous prospective studies have been conducted to further elucidate the role of thrombosis in patients with malignancy.

Thrombosis is a common complication in cancer patients, with $15 \%$ of all cancer patients developing clinically apparent thrombosis [2]. Hypercoagulability in malignancy represents a clinical spectrum ranging from abnormal coagulation tests but no clinically evident thromboembolic disease, to arteriovenous thrombosis, migratory thrombophlebitis, NBTE and disseminated intravascular coagulation [3].

NBTE, also known as marantic endocarditis, is due to uninfected vegetations on heart valves of patients with malignancy, systemic lupus erythematosus, antiphospholipid antibody syndrome or other diseases that manifest with hypercoagulability. In our case, paroxysmal nocturnal hemoglobinuria marker, protein $\mathrm{C}$, protein S, factor Leiden $\mathrm{V}$, antithrombin III, antinuclear antibody, lupus anticoagulant, erythrocyte sedimentation rate, homocysteine and vitamin B12 levels were within normal limits.

Pathogenesis of NBTE is considered to be similar to the mechanisms underlying cancer hypercoagulability. General factors, tumor-specific factors and chemotherapyrelated factors can play a role in disorders of coagulation in patients with cancer. When interacting with host cells, malignant cells can release procoagulant and fibrinolytic substances as well as inflammatory cytokines such as interleukin $1 \beta$ (IL-1 $\beta$ ), tumor necrosis factor alpha (TNF)- $\alpha$, and vascular endothelial growth factor. Inflammatory cytokines such as TNF- $\alpha$ and IL- $1 \beta$ derived from malignant cells can induce endothelial cells lining blood vessels to increase tissue factor procoagulant activity and to produce the fibrinolysis inhibitor plasminogen activator inhibitor 1 . The combination of the increase in procoagulant activity and decrease in fibrinolytic activity accelerates the prothrombotic potential of endothelial cells in malignancy [4]. NBTE as a manifestation of hypercoagulability in OCCC is likely secondary to the pathogenesis as described above.

Anticancer therapy can also increase the risk of hypercoagulability and thrombosis in cancer patients. A multivariate logistic analysis of risk factors for DVT or pulmonary embolism found that the first lifetime episode of venous thromboembolism diagnosed in the community had a 4-fold increase in cancer patients; this risk was further increased in cancer patients who had received chemotherapy or radiotherapy [5]. Mechanisms for this include the release of procoagulants and cytokines by tumor cells damaged by chemotherapy; direct damage of the vascular endothelium by chemotherapy or radiotherapy; and the reduction in plasma levels of anticoagulant proteins such as antithrombin III, protein $\mathrm{C}$ and protein $\mathrm{S}$, likely due to the direct 
hepatotoxic effects of chemoradiotherapy [4]. It has never been reported before, but the anticancer drugs carboplatin and paclitaxel, received by our patient, may have increased her risk of hypercoagulability.

NBTE vegetations are smaller and mostly detected by TEE, as in our case. Histologically, NBTE vegetations are made up of fibrin and platelets and have a very minimal inflammatory component, which can dislodge easily and cause multiple systemic embolic infarcts. As in our case, multiple bilateral renal infarcts were seen along with cerebrovascular infarcts. Cerebrovascular embolization ranges from 1491\% in NBTE [6]. NBTE most commonly affects the aortic valve, as seen in our patient. Unlike in infective endocarditis, NBTE does not destroy the cardiac valves and seldom presents with heart valve dysfunction.

Our report illustrates a patient with OCCC who presented with acute cerebrovascular events secondary to NBTE. Adenocarcinoma of the ovary has been described as a cause for NBTE, but clear cell carcinoma of the ovary presenting with NBTE is very rare [7]. Currently, there are only 2 published case reports on NBTE in clear cell carcinoma. Systemic embolization from NBTE to pulmonary, coronary, splenic and renal territories was described by Aryana et al. [6], and without pulmonary territory in Oueida and Scola [7]. However, cerebrovascular infracts in a patient with clear cell carcinoma with NBTE has not been reported in the literature thus far. Discussion of the recurrence of clear cell carcinoma and its clinical implications are beyond the scope of this paper.

Brain MRI with series including diffusion-weighted images, apparent diffusion coefficient and $\mathrm{T}_{1}$ contrast help distinguish metastases versus embolism in patients presenting with neurological deficits in malignancy. In our case, brain MRI with and without contrast showed bilateral occipital lobe restricted diffusion and edema, right greater than left, and punctuate foci of restricted diffusion in the frontal lobes, left caudate and both cerebellar hemispheres, representing embolic acute infarcts; the lack of contrast enhancement in $\mathrm{T}_{1}$ favors embolic ischemic stroke rather than metastasis.

The treatment of NBTE includes anticoagulation and appropriate treatment of underlying cancer, although there is no established strategy for anticoagulation. Anticoagulation is usually started with low-molecular-weight heparin (LMWH); unfractionated heparin is used in cases not responding to LMWH [8]. For unknown reasons, warfarin is not effective for NBTE. A study performed using heparin versus warfarin in patients with NBTE and malignancy only showed benefit with heparin [9]. In our patient, the size of the vegetation decreased after the anticoagulation with enoxaparin.

Cardiac surgery should be considered in NBTE vegetations greater than $1 \mathrm{~cm}$ with potentially curable cancer and avoided in those with advanced non-curable malignancies because surgery cannot prevent further formation and embolization of NBTE. Our patient was not considered for cardiac surgery as she had advanced metastases to the liver, mesentery and vertebra. She was placed on an IVC filter and started on long-term anticoagulation with a therapeutic dose of enoxaparin for treatment of her pulmonary embolism, DVT and NBTE.

NBTE can occur at any stage of malignancy, but is commonly seen in the advanced stages. Sometimes it can herald the advancement of the malignancy, as in our patient 
who initially presented with stage I OCCC, but was found to have advanced malignancy on further workup after detection of NBTE.

Morbidity and mortality from embolization can be reduced by early identification of NBTE and appropriate anticoagulation. Thus far, there are no specific guidelines regarding appropriate screening and management of NBTE in malignancy. Some case review series in the literature guide current clinical management.

\section{Conclusion}

This case has been reported because of the rare and unique association of OCCC, NBTE and embolic stroke. NBTE is a rare manifestation of malignancy and is routinely underevaluated. Morbidity and mortality from embolization can be reduced by early identification of NBTE and appropriate anticoagulation. In view of the very limited data on NBTE in OCCC, our case report can be used as a tool in formulating guidelines for early diagnosis and management.

\section{Disclosure Statement}

The authors declare that they have no competing interests.
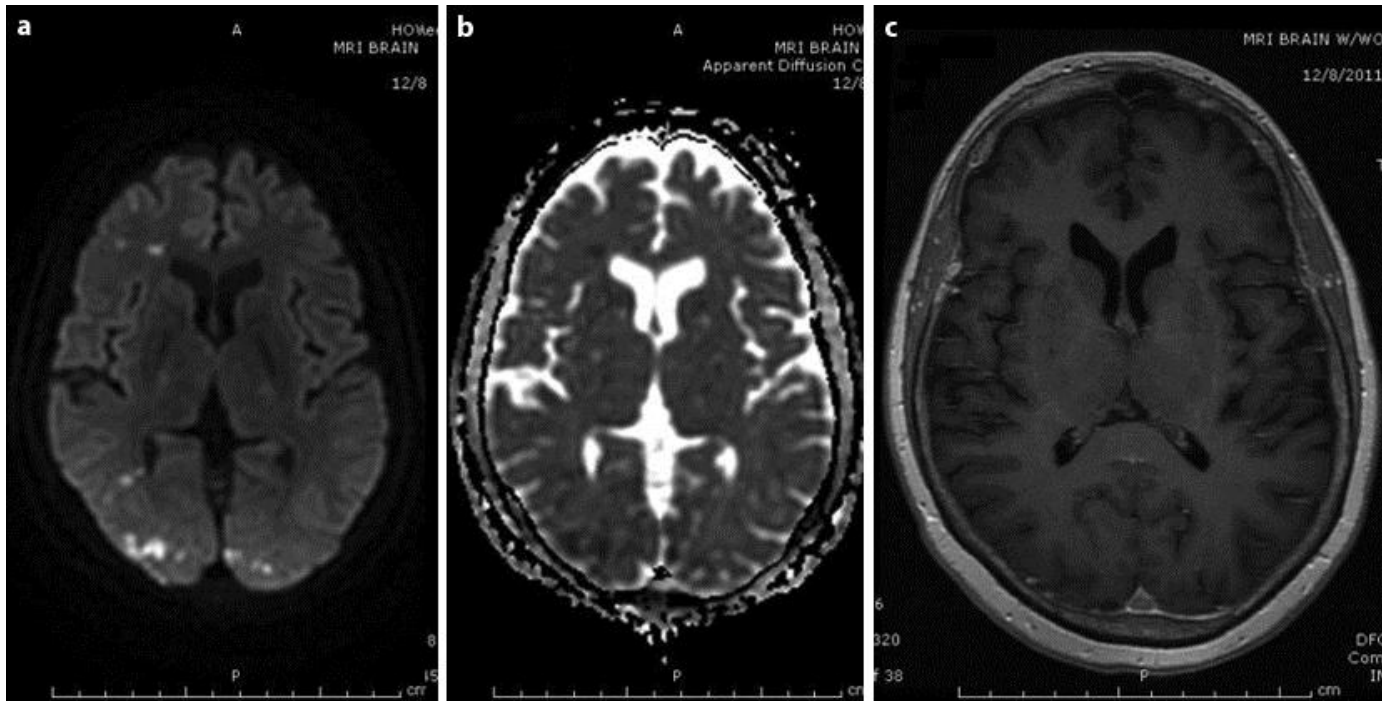

Fig. 1. a, b Brain MRI showing bilateral restricted diffusion and edema in the occipital lobes (right > left) and punctuate foci in the right frontal lobe representing acute infarct. $\mathbf{c}$ Brain MRI, axial $\mathrm{T}_{1}$ postcontrast image showing no enhancement in the infarcted area. 

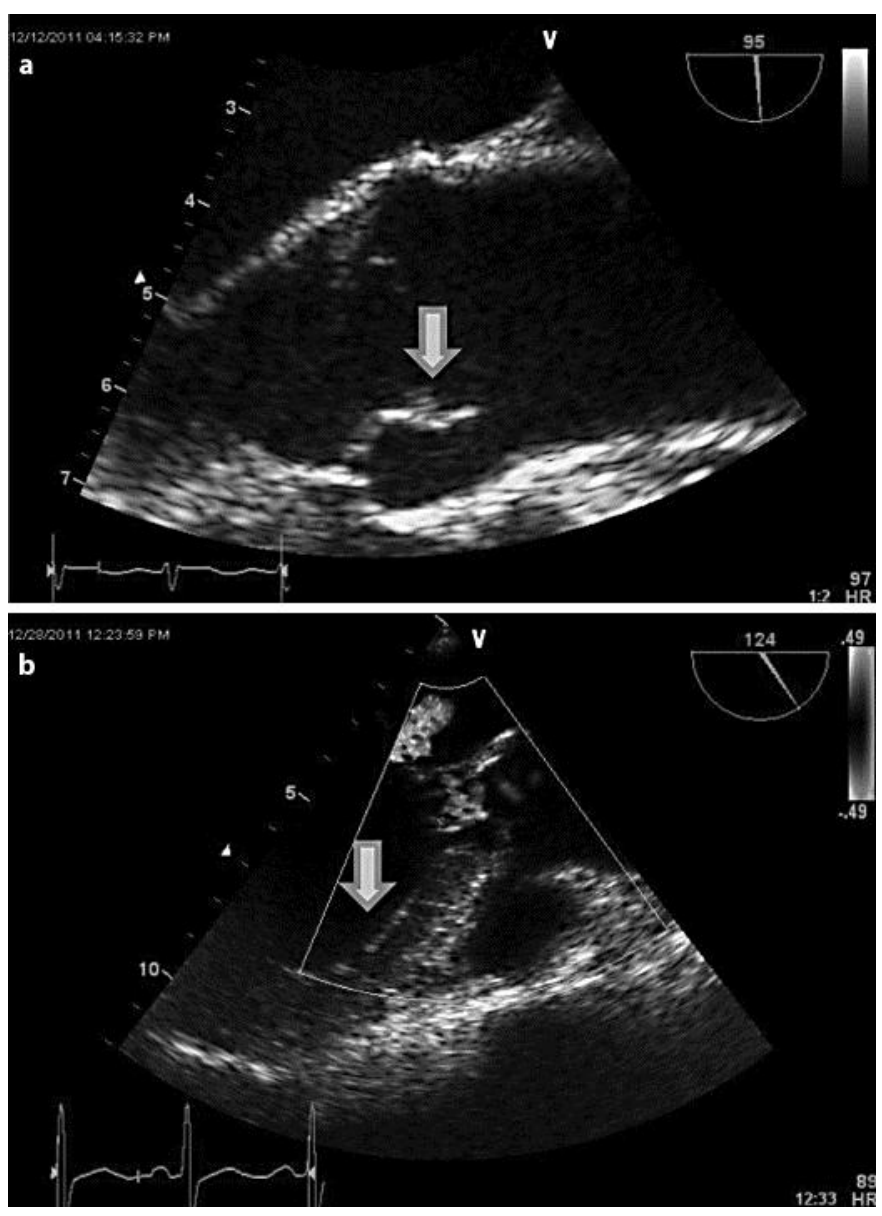

Fig. 2. a TEE showing small $(7 \times 2 \mathrm{~mm})$ mobile linear echodensity on the aortic side of the right aortic valve cusp, consistent with vegetation. $\mathbf{b}$ Repeat TEE after the anticoagulation showing the decrease in the size $(2 \mathrm{~mm})$ of the vegetation. 


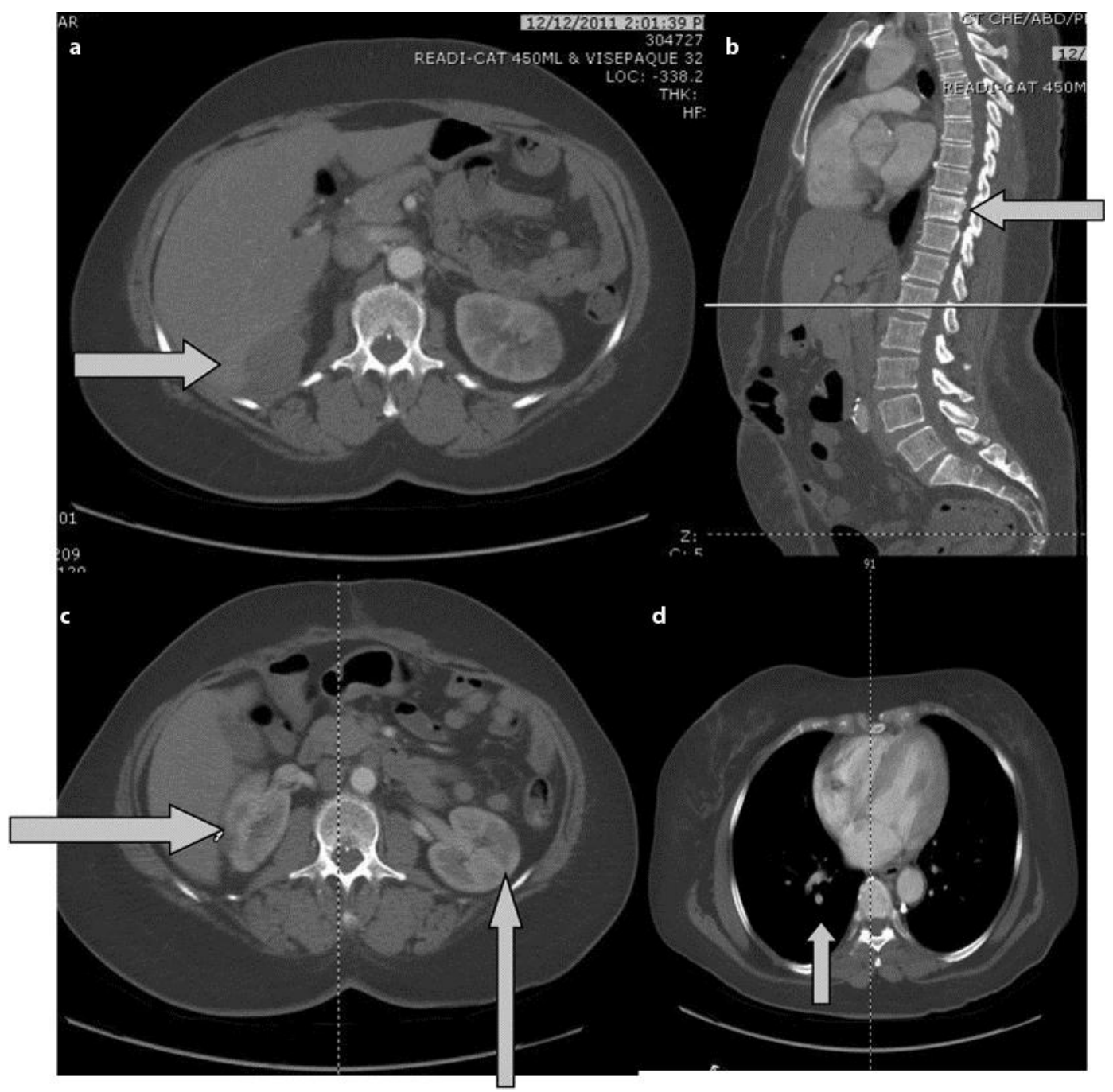

Fig. 3. a, b Abdominal CT showing the metastases to the liver, and T10 vertebral body. c Abdominal CT showing the multiple wedge-shaped infarcts in both kidneys. $\mathbf{d}$ Chest CT showing an acute pulmonary embolus in the subsegmental artery of the right lower lung lobe. 


\section{References}

1 Chaturvedi S, Ansell J, Recht L: Should cerebral ischemic events in cancer patients be considered a manifestation of hypercoagulability? Stroke 1994;25:1215-1218.

72 Rickles FR, Levine MN: Epidemiology of thrombosis in cancer. Acta Haematol 2001;106:6-12.

3 Hussain S: Thrombophilia in malignancy: a review of the literature. Internet J Int Med 2009;8.

-4 Falanga A, Donati MB: Pathogenesis of thrombosis in patients with malignancy. Int J Hematol 2001;73:137-144.

5 Heit JA, Silverstein MD, Mohr DN, Petterson TM, O’Fallon WM, Melton LJ 3rd: Risk factors for deep vein thrombosis and pulmonary embolism: a population-based case-control study. Arch Intern Med 2000;160:809.

-6 Aryana A, Esterbrooks DJ, Morris PC: Nonbacterial thrombotic endocarditis with recurrent embolic events as manifestation of ovarian neoplasm. J Gen Intern Med 2006;21:C12-C15.

7 Oueida Z, Scola M: Ovarian clear cell carcinoma presenting as non-bacterial thrombotic endocarditis and systemic embolization. World J Oncol 2011;2:270-274.

-8 El-Shami K, Griffiths E, Streiff M: Nonbacterial thrombotic endocarditis in cancer patients: pathogenesis, diagnosis, and treatment. Oncologist 2007;12:518-523.

$\checkmark 9$ Salem DN, Stein PD, Al-Ahmad A, Bussey HI, Horstkotte D, Miller N, et al: Antithrombotic therapy in valvular heart disease - native and prosthetic: the Seventh ACCP Conference on Antithrombotic and Thrombolytic Therapy. Chest 2004;126(3 suppl):457S-482S 